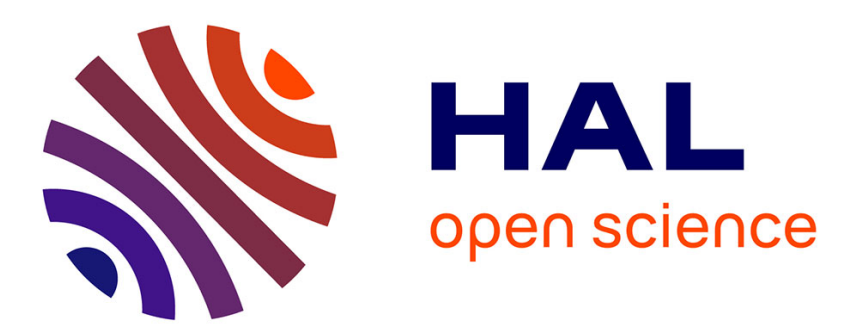

\title{
Every ordinary differential equation with a strict Lyapunov function is a gradient system
}

Tomáš Bárta, Ralph Chill, Eva Fašangová

\section{To cite this version:}

Tomáš Bárta, Ralph Chill, Eva Fašangová. Every ordinary differential equation with a strict Lyapunov function is a gradient system. Monatshefte für Mathematik, 2012, 166 (1), pp.57-72. 10.1007/s00605011-0322-4 . hal-01275665

\section{HAL Id: hal-01275665 \\ https://hal.science/hal-01275665}

Submitted on 17 Feb 2016

HAL is a multi-disciplinary open access archive for the deposit and dissemination of scientific research documents, whether they are published or not. The documents may come from teaching and research institutions in France or abroad, or from public or private research centers.
L'archive ouverte pluridisciplinaire HAL, est destinée au dépôt et à la diffusion de documents scientifiques de niveau recherche, publiés ou non, émanant des établissements d'enseignement et de recherche français ou étrangers, des laboratoires publics ou privés. 


\title{
Every ordinary differential equation with a strict Lyapunov function is a gradient system
}

\author{
Tomáš Bárta · Ralph Chill · Eva \\ Fašangová
}

Received: date / Accepted: date

\begin{abstract}
We explain and prove the statement from the title. This allows us to formulate a new type of gradient inequality and to obtain a new stabilization result for gradient-like ordinary differential equations.
\end{abstract}

Keywords Strict Lyapunov function · gradient system · Kurdyka-Łojasiewicz gradient inequality · convergence to equilibrium · damped second order ordinary differential equation

Mathematics Subject Classification (2000) 37B25 - 34D05 - 34C40

\section{Introduction}

The theory of Lyapunov functions is a fundamental part of the stability theory of ordinary differential equations [14], [3], because many ordinary differential equations naturally admit an energy function which is nonincreasing along solutions. Moreover, La Salle's invariance principle [13], [3, Chapter VIII], [8] and gradient inequalities for the underlying Lyapunov function [15], [11], [9],

The first author was supported by the grant MSM 0021620839 from the Czech Ministry of Education.

Tomáš Bárta

Department of Mathematical Analysis, Charles University, Sokolovská 83, 18675 Praha 8, Czech Republic E-mail: barta@karlin.mff.cuni.cz

Ralph Chill

Université Paul Verlaine - Metz, Laboratoire de Mathématiques et Applications de Metz et CNRS, UMR 7122, Bât. A, Ile du Saulcy, 57045 Metz Cedex 1, France E-mail: chill@univmetz.fr

Eva Fašangová

Department of Mathematical Analysis, Charles University, Sokolovská 83, 18675 Praha 8, Czech Republic and Institut für Angewandte Analysis, Universität Ulm, 89069 Ulm, Germany E-mail: fasanga@karlin.mff.cuni.cz 
[1], [12], [5] provide easy and powerful stabilization results.

Let $F$ be a continuous tangent vector field on a manifold $M$. Following [8], we say that a continuously differentiable function $\mathcal{E}: M \rightarrow \mathbb{R}$ is a strict Lyapunov function for the ordinary differential equation

$$
\dot{u}+F(u)=0,
$$

if

$$
\left\langle\mathcal{E}^{\prime}(u), F(u)\right\rangle>0 \quad \text { whenever } u \in M \text { and } F(u) \neq 0 .
$$

Every strict Lyapunov function is nonincreasing along solutions of (1), and if it is constant on some solution then that solution must be stationary. Among ordinary differential equations, the gradient systems on a Riemannian manifold $(M, g)$

$$
\dot{u}+\nabla_{g} \mathcal{E}(u)=0
$$

are the prototype examples of dissipative systems which admit a strict Lyapunov function: namely the function $\mathcal{E}$ itself. In Theorem 1 we observe the following simple converse result, announced in the title: every ordinary differential equation (1) with a strict Lyapunov function is - on the open subset of nonequilibrium points of $F$ - a gradient system. More precisely, it is a gradient system for the Lyapunov function itself and for an appropriate Riemannian metric. This observation, which may be deduced from [16], [17] but which was formulated differently there, implies that the gradient systems are basically the only examples of dissipative systems with strict Lyapunov function. As an application, we obtain that the damped second order equation

$$
\ddot{u}+G(\dot{u})+\nabla E(u)=0
$$

is a gradient system when it is rewritten as a first order equation.

We apply this result in order to obtain a stabilization result for global solutions of the general system (1) (Theorem 3). Strictly speaking, this result can be and actually is formulated independently of the gradient system structure in the appropriate ambient metric constructed in Theorem 1. However, it can be better motivated with this knowledge behind.

The classical Łojasiewicz gradient inequality (see [15])

$$
|\mathcal{E}(v)-\mathcal{E}(\varphi)|^{1-\theta} \leq C\left\|\mathcal{E}^{\prime}(v)\right\|_{g}
$$

and the more general Kurdyka-Łojasiewicz gradient inequality (see [11], and [4], [5] for the name)

$$
\Theta(|\mathcal{E}(v)-\mathcal{E}(\varphi)|) \leq\left\|\mathcal{E}^{\prime}(v)\right\|_{g}
$$

have turned out to be particularly useful for proving that global solutions of gradient systems converge to a single equilibrium. The first convergence result for gradient systems is due to Lojasiewicz himself [15]; he also proved that 
every real analytic function satisfies the Łojasiewicz gradient inequality (4). Later, Haraux \& Jendoubi and Alvarez et al. proved convergence results for damped second order ordinary differential equations by using a gradient inequality for a natural Lyapunov function [9], [2]. More recently, it has been shown that many convergence results can be unified into a general theorem for the ordinary differential equation (1), provided that that equation admits a strict Lyapunov function, the Lyapunov function satisfies a gradient inequality and an angle condition holds between $\mathcal{E}^{\prime}$ and $F$ [1], [12], [7]. This angle condition is automatically satisfied for gradient systems but there are interesting examples where it is not satisfied (see [6] and Section 5 below).

Now, if the problem of convergence to equilibrium - with respect to some given metric - is approached by knowing that the problem (1) has a gradient structure on the open subset of nonequilibrium points - with respect to a different metric -, then one is naturally led to the problem of comparing the two involved metrics. This problem is even crucial since, by La Salle's invariance principle, the only possible limit points of solutions are equilibrium points, and since equilibrium points lie on the boundary of the set of nonequilibrium points. The comparison of metrics motivates us to formulate the gradient inequality

$$
\Theta(|\mathcal{E}(v)-\mathcal{E}(\varphi)|) \leq\left\langle\mathcal{E}^{\prime}(v), \frac{F(v)}{\|F(v)\|_{g}}\right\rangle
$$

and a convergence result (Theorem 3) which generalizes the results from [1], $[12],[7]$ for ordinary differential equations. With this view we can also give a new interpretation of a recent convergence result by Chergui [6] for a second order ordinary differential equation with degenerate damping.

Of course, if the original and new metric are equivalent, then the induced distance functions are equivalent because lengthes of curves are equivalent. Since length is an important notion in the above cited convergence results, we characterize equivalence of the two metrics. We find that the two metrics are equivalent if and only if an angle condition and a comparability condition between $\mathcal{E}^{\prime}$ and $F$ holds (Theorem 2). These are exactly the conditions which appear in [1], [12], [7]. Finally, we formulate some open questions concerning the metric with respect to which equation (1) is a gradient system.

\section{Main result}

Before stating the main theorem, let us fix some notation. When we write manifold in this article, we mean a differentiable and finite-dimensional manifold. The duality between tangent vectors and cotangent vectors is denoted by $\langle\cdot, \cdot\rangle$. Whenever $g$ is a Riemannian metric, we write $\langle\cdot, \cdot\rangle_{g}$ for the inner product on the tangent space; to be precise, we should write $\langle\cdot, \cdot\rangle_{g(u)}$ for the inner product on the tangent space $T_{u} M$, but the variable $u$ is dropped, and equalities and inequalities are usually to be understood for functions on $M$. We write $\|\cdot\|_{g}$ 
for the induced norm both on the tangent space and on the cotangent space. Given a differentiable function $\mathcal{E}: M \rightarrow \mathbb{R}$, we denote by $\mathcal{E}^{\prime}$ its derivative. The derivative $\mathcal{E}^{\prime}$ is a cotangent vector field; the associated, uniquely determined tangent vector field $\nabla_{g} \mathcal{E}$, given by

$$
\left\langle\mathcal{E}^{\prime}, X\right\rangle=\left\langle\nabla_{g} \mathcal{E}, X\right\rangle_{g} \text { for every tangent vector field } X,
$$

is called the gradient of $\mathcal{E}$ with respect to the metric $g$.

The following is the theorem announced in the title of this article. It should be compared to [16, Theorem 1], [17, Proposition 2.8], where it was stated that if the ordinary differential equation (1) (in $\mathbb{R}^{N}$ ) admits a strict Lyapunov function $\mathcal{E}$, then $F=L \nabla \mathcal{E}$, where $L$ is a continuous function taking its values in the positive definite matrices and $\nabla \mathcal{E}$ is the Euclidean gradient. Note that the multiplication by $L$ corresponds to a change of the underlying metric.

Theorem 1 Let $M$ be a manifold, $F$ a continuous tangent vector field on $M$, and let $\mathcal{E}: M \rightarrow \mathbb{R}$ be a continuously differentiable, strict Lyapunov function for (1). Then there exists a Riemannian metric $\tilde{g}$ on the open set

$$
\tilde{M}:=\{u \in M: F(u) \neq 0\} \subseteq M
$$

such that $\nabla_{\tilde{g}} \mathcal{E}=F$. In particular, the differential equation (1) is a gradient system on the Riemannian manifold $(\tilde{M}, \tilde{g})$.

Proof By assumption (2), $0 \neq F(u) \notin \operatorname{ker} \mathcal{E}^{\prime}(u)$ and $\operatorname{ker} \mathcal{E}^{\prime}(u) \neq T_{u} M$ for every $u \in \tilde{M}$. As a consequence the tangent bundle $T \tilde{M}$ is the direct sum of the bundle $\operatorname{ker} \mathcal{E}^{\prime}$ and the bundle generated by the vector field $F$ :

$$
T \tilde{M}=\operatorname{ker} \mathcal{E}^{\prime} \oplus\langle F\rangle .
$$

For every continuous tangent vector field $X$ on $\tilde{M}$, the vector fields

$$
X_{0}:=X-\frac{\left\langle\mathcal{E}^{\prime}, X\right\rangle}{\left\langle\mathcal{E}^{\prime}, F\right\rangle} F \text { and } X_{1}:=\frac{\left\langle\mathcal{E}^{\prime}, X\right\rangle}{\left\langle\mathcal{E}^{\prime}, F\right\rangle} F
$$

are well-defined and continuous, $X_{0} \in \operatorname{ker} \mathcal{E}^{\prime}$ and $X_{1} \in\langle F\rangle$ (the one-dimensional space - or vector bundle - generated by $F$ ). Hence, the above decomposition of the tangent space $T \tilde{M}$ is continuous in the sense that the projection onto $\operatorname{ker} \mathcal{E}^{\prime}$ along $\langle F\rangle$ is a continuous function.

Now we choose an arbitrary Riemannian metric $g$ on $M$. Starting from this metric, we define a new metric on $\tilde{M}$ by setting

$$
\begin{aligned}
\langle X, Y\rangle_{\tilde{g}} & :=\left\langle X_{0}, Y_{0}\right\rangle_{g}+\frac{1}{\left\langle\mathcal{E}^{\prime}, F\right\rangle}\left\langle\mathcal{E}^{\prime}, X\right\rangle\left\langle\mathcal{E}^{\prime}, Y\right\rangle \\
& =\left\langle X_{0}, Y_{0}\right\rangle_{g}+\frac{1}{\left\langle\mathcal{E}^{\prime}, F\right\rangle}\left\langle\mathcal{E}^{\prime}, X_{1}\right\rangle\left\langle\mathcal{E}^{\prime}, Y_{1}\right\rangle .
\end{aligned}
$$

Precisely at this point we use the assumption that $\mathcal{E}$ is a strict Lyapunov function, that is, $\left\langle\mathcal{E}^{\prime}, F\right\rangle>0$ on $\tilde{M}$, because this assumption implies that $\tilde{g}$ 
really is a metric (in particular: positive definite). Since the decomposition (6) is continuous, and since $g, \mathcal{E}^{\prime}$ and $F$ are continuous, $\tilde{g}$ is continuous, too.

By definition of the metric $\tilde{g}$ and by definition of the gradient $\nabla_{\tilde{g}} \mathcal{E}$, we have for every tangent vector field $X$

$$
\langle F, X\rangle_{\tilde{g}}=0+\left\langle\mathcal{E}^{\prime}, X\right\rangle=\left\langle\nabla_{\tilde{g}} \mathcal{E}, X\right\rangle_{\tilde{g}}
$$

so that $F=\nabla_{\tilde{g}} \mathcal{E}$ and the claim is proved.

Remark 1 Note carefully that the metric $\tilde{g}$, for which we have the equality $F=\nabla_{\tilde{g}} \mathcal{E}$, is not unique. The inner product $\langle X, Y\rangle_{\tilde{g}}$ is uniquely determined by the functions $F, \mathcal{E}$ if one of the two vectors $X$ or $Y$ is a multiple of $F$; this fact comes from the requirement that one wants to have $F=\nabla_{\tilde{g}} \mathcal{E}$. However, on $\operatorname{ker} \mathcal{E}^{\prime} \times \operatorname{ker} \mathcal{E}^{\prime}$ one has a free choice, how to define the metric $\tilde{g}$.

Theorem 1 says that equation (1) is a gradient system on $(\tilde{M}, \tilde{g})$. The set $\tilde{M}$ being a subset of $M$ and the metric $\tilde{g}$ being a new metric, several questions arise.

Question 1 When is the equation (1) a gradient system on the whole of $M$ ?

We ask this question in a slightly different way and in weaker forms. Recall from Remark 1 above that the metric $\tilde{g}$ from Theorem 1 is not uniquely determined. If we ask whether the ordinary differential equation (1) is a gradient system associated with the fixed Lyapunov function $\mathcal{E}$, then Question 1 is closely related to the following one.

Question 2 Can we choose the metric $\tilde{g}$ such that $\tilde{g}$ extends to a Riemannian metric on $M$ ?

Example 1 (Not every metric $\tilde{g}$ extends to a Riemannian metric on $M$ ) Let $M=\mathbb{R}^{2}$, and let $F: \mathbb{R}^{2} \rightarrow \mathbb{R}^{2}$ and $\mathcal{E}: \mathbb{R}^{2} \rightarrow \mathbb{R}$ be given by

$$
F\left(u_{1}, u_{2}\right):=\left(u_{1}, 2 u_{2}\right) \quad \text { and } \quad \mathcal{E}\left(u_{1}, u_{2}\right):=\frac{1}{2}\left(u_{1}^{2}+u_{2}^{2}\right)
$$

The origin $u=(0,0)$ is the only equilibrium point of $F$ and for $u \neq(0,0)$ we have

$$
\left\langle\mathcal{E}^{\prime}(u), F(u)\right\rangle=u_{1}^{2}+2 u_{2}^{2}>0 .
$$

Hence, $\mathcal{E}$ is a strict Lyapunov function.

Let $g$ be the Euclidean metric, and define the new metric $\tilde{g}$ by using the formula (7), that is, for every $u, X, Y \in \mathbb{R}^{2}$,

$$
\begin{aligned}
\langle X, Y\rangle_{\tilde{g}(u)}:=\left\langle X-\frac{\left\langle\mathcal{E}^{\prime}(u), X\right\rangle}{\left\langle\mathcal{E}^{\prime}(u), F(u)\right\rangle} F(u), Y-\frac{\left\langle\mathcal{E}^{\prime}(u), Y\right\rangle}{\left\langle\mathcal{E}^{\prime}(u), F(u)\right\rangle} F(u)\right\rangle_{g(u)}+ \\
+\frac{1}{\left\langle\mathcal{E}^{\prime}(u), F(u)\right\rangle}\left\langle\mathcal{E}^{\prime}(u), X\right\rangle\left\langle\mathcal{E}^{\prime}(u), Y\right\rangle .
\end{aligned}
$$


Then, for $X=(1,0)$ and $Y=(0,1)$ a straightforward calculation shows that

$$
\lim _{h \rightarrow 0}\langle X, Y\rangle_{\tilde{g}(0, h)}=0 \quad \text { and } \quad \lim _{h \rightarrow 0}\langle X, Y\rangle_{\tilde{g}(h, h)}=-\frac{1}{9} .
$$

Hence, the metric $\tilde{g}$ does not have a continuous extension at the origin.

Now, let $g$ be the metric given by

$$
\langle X, Y\rangle_{g}=X_{1} Y_{1}+\frac{1}{2} X_{2} Y_{2},
$$

and define $\tilde{g}$ starting with this metric $g$. Then $\tilde{g}$ does have a continuous extension near the origin. In fact, in this case $\tilde{g}=g$.

\section{Equivalence of metrics}

Let $g$ be any Riemannian metric on $M$ and let $\tilde{g}$ be the Riemannian metric on $\tilde{M}$ constructed from $g$ as in the proof of Theorem 1 . If the metric $\tilde{g}$ admits a continuous extension to $M$, then $g$ and $\tilde{g}$ are locally equivalent on $\tilde{M}$ (and even on $M$ ). The converse is not true in general (take the metrics from Example 1). In this section, we characterize equivalence of the two metrics $g$ and $\tilde{g}$ on $\tilde{M}$ in terms of an angle condition which has recently appeared in some articles on stabilization of gradient-like systems [1], [12], [7] and in terms of a comparability condition.

We say that two Riemannian metrics $g_{1}, g_{2}$ on a manifold $M$ are equivalent, if there exist $c_{1}, c_{2}>0$ such that for every tangent vector field $X$

$$
c_{1}\|X\|_{g_{1}} \leq\|X\|_{g_{2}} \leq c_{2}\|X\|_{g_{1}} \text { on } M
$$

Note that if two Riemannian metrics are equivalent then the induced distance functions are equivalent, too. In particular, the completions of $\left(M, g_{1}\right)$ and $\left(M, g_{2}\right)$ are the same and the boundaries in this completion carry the same topology.

We say that $\mathcal{E}^{\prime}$ and $F$ satisfy the angle condition (see [1, Condition (2.2)], [12, Condition (AC), Definition 1.1], [7, Conditions (4), (9)]) if there exists $\alpha>0$ such that

$$
\left\langle\mathcal{E}^{\prime}, F\right\rangle \geq \alpha\left\|\mathcal{E}^{\prime}\right\|_{g}\|F\|_{g} \text { on } \tilde{M},
$$

and we say that they satisfy the comparability condition if there exist constants $c_{1}, c_{2}>0$ such that

$$
c_{1}\left\|\mathcal{E}^{\prime}\right\|_{g} \leq\|F\|_{g} \leq c_{2}\left\|\mathcal{E}^{\prime}\right\|_{g} \text { on } \tilde{M}
$$

It is straightforward to check that the angle condition and the comparability condition together are equivalent to the existence of a constant $\beta>0$ such that

$$
\left\langle\mathcal{E}^{\prime}, F\right\rangle \geq \beta\left(\left\|\mathcal{E}^{\prime}\right\|_{g}^{2}+\|F\|_{g}^{2}\right) \text { on } \tilde{M}
$$

this condition appears in various examples in [7, Section 2]. 
Theorem 2 The metrics $g$ and $\tilde{g}$ are equivalent on $\tilde{M}$ if and only if $\mathcal{E}^{\prime}$ and $F$ satisfy the conditions (AC) and $(\mathrm{C})$.

Proof Assume first that the two metrics $g$ and $\tilde{g}$ are equivalent. Then there exist constants $c_{1}, c_{2}>0$ such that for every tangent vector field $X$

$$
c_{1}\|X\|_{g} \leq\|X\|_{\tilde{g}} \leq c_{2}\|X\|_{g} \text { on } \tilde{M} .
$$

Then

$$
\left\|\mathcal{E}^{\prime}\right\|_{\tilde{g}}=\sup _{\|X\|_{\tilde{g} \leq 1}}\left\langle\mathcal{E}^{\prime}, X\right\rangle \geq \sup _{c_{2}\|X\|_{g} \leq 1}\left\langle\mathcal{E}^{\prime}, X\right\rangle=\frac{1}{c_{2}}\left\|\mathcal{E}^{\prime}\right\|_{g} \text { on } \tilde{M} .
$$

Since $F=\nabla_{\tilde{g}} \mathcal{E}$, the preceding inequalities imply

$$
\left\langle\mathcal{E}^{\prime}, F\right\rangle=\left\langle\nabla_{\tilde{g}} \mathcal{E}, F\right\rangle_{\tilde{g}}=\left\|\nabla_{\tilde{g}} \mathcal{E}\right\|_{\tilde{g}}\|F\|_{\tilde{g}}=\left\|\mathcal{E}^{\prime}\right\|_{\tilde{g}}\|F\|_{\tilde{g}} \geq \frac{c_{1}}{c_{2}}\left\|\mathcal{E}^{\prime}\right\|_{g}\|F\|_{g},
$$

that is, $\mathcal{E}^{\prime}$ and $F$ satisfy the angle condition $(\mathrm{AC})$. Moreover,

$$
\|F\|_{\tilde{g}}=\left\|\nabla_{\tilde{g}} \mathcal{E}\right\|_{\tilde{g}}=\left\|\mathcal{E}^{\prime}\right\|_{\tilde{g}}
$$

and the equivalence of $g$ and $\tilde{g}$ implies also the comparability condition (C).

Now assume that $\mathcal{E}^{\prime}$ and $F$ satisfy the angle and comparability conditions (AC) and (C). Given a tangent vector field $X$, we use the decomposition (6), that is, $X=X_{0}+X_{1}$ with $X_{0} \in \operatorname{ker} \mathcal{E}^{\prime}$ and $X_{1} \in\langle F\rangle$. We have,

$$
\begin{aligned}
\|X\|_{\tilde{g}}^{2} & =\left\|X_{0}\right\|_{g}^{2}+\frac{\left\langle\mathcal{E}^{\prime}, X_{1}\right\rangle^{2}}{\left\langle\mathcal{E}^{\prime}, F\right\rangle} & & \text { (definition of } \tilde{g}) \\
& \geq\left\|X_{0}\right\|_{g}^{2}+\frac{\alpha^{2}\left\|\mathcal{E}^{\prime}\right\|_{g}^{2}\left\|X_{1}\right\|_{g}^{2}}{\left\|\mathcal{E}^{\prime}\right\|_{g}\|F\|_{g}} & & (\text { by }(A C) \text { and Cauchy-Schwarz) } \\
& \geq\left\|X_{0}\right\|_{g}^{2}+\frac{\alpha^{2}}{c_{2}}\left\|X_{1}\right\|_{g}^{2} & & (\text { by }(C)) \\
& \geq \frac{1}{2} \min \left\{1, \frac{\alpha^{2}}{c_{2}}\right\}\|X\|_{g}^{2} & & \text { (triangle inequality), }
\end{aligned}
$$

and

$$
\begin{array}{rlrl}
\|X\|_{\tilde{g}}^{2} & =\left\|X_{0}\right\|_{g}^{2}+\frac{\left\langle\mathcal{E}^{\prime}, X\right\rangle^{2}}{\left\langle\mathcal{E}^{\prime}, F\right\rangle} & & \text { (definition of } \tilde{g}) \\
& \leq 2\|X\|_{g}^{2}+2\left\|X_{1}\right\|_{g}^{2}+\frac{\left\langle\mathcal{E}^{\prime}, X\right\rangle^{2}}{\left\langle\mathcal{E}^{\prime}, F\right\rangle} & & \text { (triangle inequality) } \\
& =2\|X\|_{g}^{2}+2 \frac{\left\langle\mathcal{E}^{\prime}, X\right\rangle^{2}}{\left\langle\mathcal{E}^{\prime}, F\right\rangle^{2}}\|F\|_{g}^{2}+\frac{\left\langle\mathcal{E}^{\prime}, X\right\rangle^{2}}{\left\langle\mathcal{E}^{\prime}, F\right\rangle} & & \text { (definition of } \left.X_{1}\right) \\
& \leq 2\|X\|_{g}^{2}+\frac{2}{\alpha^{2}}\|X\|_{g}^{2}+\frac{\left\|\mathcal{E}^{\prime}\right\|_{g}^{2}\|X\|_{g}^{2}}{\alpha\left\|_{\mathcal{E}^{\prime}}\right\|_{g}\|F\|_{g}} & & \text { (by Cauchy-Schwarz and }(A C)) \\
& \leq 2\|X\|_{g}^{2}+\frac{2}{\alpha^{2}}\|X\|_{g}^{2}+\frac{1}{\alpha c_{1}}\|X\|_{g}^{2} & & (\text { by }(C)) \\
& =\left(2+\frac{2}{\alpha^{2}}+\frac{1}{\alpha c_{1}}\right)\|X\|_{g}^{2}, &
\end{array}
$$

that is, $g$ and $\tilde{g}$ are equivalent. 


\section{Asymptotics}

In this section, $(M, g)$ is a Riemannian manifold. We assume that the ordinary differential equation (1) with initial condition $u(0)=u_{0} \in M$ is uniquely solvable. Given a function $u: \mathbb{R}_{+} \rightarrow M$, the set of all accumulation points (as $t \rightarrow \infty)$

$$
\omega(u)=\left\{\varphi \in M: \text { there exists }\left(t_{n}\right) \nearrow \infty \text { such that } u\left(t_{n}\right) \rightarrow \varphi\right\}
$$

is called the $\omega$-limit set of $u$.

Theorem 3 Let $F$ be a continuous tangent vector field on the Riemannian manifold $(M, g)$. Let $u: \mathbb{R}_{+} \rightarrow M$ be a global solution of the ordinary differential equation (1) and let $\mathcal{E}: M \rightarrow \mathbb{R}$ be a continuously differentiable, strict Lyapunov function for (1). Assume that there exist $\Theta: \mathbb{R}_{+} \rightarrow \mathbb{R}_{+}$such that $1 / \Theta \in L_{l o c}^{1}([0,+\infty))$ and $\Theta(s)>0$ for $s>0, \varphi \in \omega(u)$ and a neighbourhood $U \subseteq M$ of $\varphi$ such that for every $v \in U \cap \tilde{M}$

$$
\Theta(|\mathcal{E}(v)-\mathcal{E}(\varphi)|) \leq\left\langle\mathcal{E}^{\prime}(v), \frac{F(v)}{\|F(v)\|_{g}}\right\rangle .
$$

Then $u$ has finite length in $(M, g)$ and, in particular, $\lim _{t \rightarrow+\infty} u(t)=\varphi$ in $(M, g)$.

Proof Since $\mathcal{E}$ is a Lyapunov function, necessarily the function $\mathcal{E}(u)$ is nonincreasing. Hence, $\lim _{t \rightarrow \infty} \mathcal{E}(u(t))$ exists in $\mathbb{R} \cup\{-\infty\}$. By continuity of the function $\mathcal{E}$ and by definition of the $\omega$-limit set $\omega(u)$, this limit equals $\mathcal{E}(\psi)$ for every $\psi \in \omega(u)$ (so, since $\omega(u)$ is non-empty by assumption, the limit of $\mathcal{E}(u)$ is finite and equals $\mathcal{E}(\varphi)$ ). By changing $\mathcal{E}$ by an additive constant, if necessary, we may without loss of generality assume that $\mathcal{E}(\varphi)=0$, so that $\mathcal{E}(u(t)) \geq 0$ and $\lim _{t \rightarrow \infty} \mathcal{E}(u(t))=0$.

If $\mathcal{E}\left(u\left(t_{0}\right)\right)=0$ for some $t_{0} \geq 0$, then $\mathcal{E}(u(t))=0$ for every $t \geq t_{0}$, and therefore, since $\mathcal{E}$ is a strict Lyapunov function, the function $u$ is constant for $t \geq t_{0}$. In this case, there remains nothing to prove.

Hence, we may assume that $\mathcal{E}(u(t))>0$ for every $t \geq 0$. By unique solvability of (1) this implies that $u(t) \in \tilde{M}$ for every $t \geq 0$. Let $\sigma>0$ be such that the closed ball $\bar{B}(\varphi, \sigma)$ (with respect to the distance $d$ induced by $g$ ) is contained in $U$. Let

$$
\Phi(t):=\int_{0}^{t} \frac{1}{\Theta(s)} \mathrm{d} s,
$$

and let $t_{0} \geq 0$ be so large that

$$
d\left(u\left(t_{0}\right), \varphi\right) \leq \frac{\sigma}{3} \text { and } \Phi\left(\mathcal{E}\left(u\left(t_{0}\right)\right)\right)<\frac{\sigma}{3} .
$$

Let

$$
t_{1}:=\inf \left\{t \geq t_{0}: d(u(t), \varphi)=\sigma\right\}
$$


By continuity of the function $u$, we have $t_{1}>t_{0}$. Then for every $t \in\left[t_{0}, t_{1}\right)$,

$$
\begin{aligned}
-\frac{d}{d t} \Phi(\mathcal{E}(u(t))) & =\frac{1}{\Theta(\mathcal{E}(u(t))}\left(-\frac{d}{d t} \mathcal{E}(u(t))\right) & & \text { (chain rule and def. of } \Phi) \\
& =\frac{1}{\Theta(\mathcal{E}(u(t))}\left\langle\mathcal{E}^{\prime}(u(t)), F(u(t))\right\rangle & & \text { (chain rule and (1)) } \\
& \geq\|F(u(t))\|_{g} & & \text { (gradient inequality }(10)) \\
& =\| \dot{u}(t)) \|_{g} & & \text { (equation }(1)) .
\end{aligned}
$$

Hence, for every $t \in\left[t_{0}, t_{1}\right)$,

$$
\begin{aligned}
d(u(t), \varphi) & \leq d\left(u(t), u\left(t_{0}\right)\right)+d\left(u\left(t_{0}\right), \varphi\right) & & \text { (triangle inequality) } \\
& \leq \int_{t_{0}}^{t}\|\dot{u}(s)\|_{g} d s+d\left(u\left(t_{0}\right), \varphi\right) & & \text { (def. of distance) } \\
& \leq \Phi\left(\mathcal{E}\left(u\left(t_{0}\right)\right)\right)+d\left(u\left(t_{0}\right), \varphi\right) & & \text { (preceding estimates) } \\
& \leq \frac{2}{3} \sigma & & \text { (choice of } \left.t_{0}\right) .
\end{aligned}
$$

This inequality implies that $t_{1}=\infty$. But then $\|\dot{u}\|_{g} \in L^{1}\left(\mathbb{R}_{+}\right)$, by the estimates (11). This means that $u$ has finite length in $(M, g)$. The existence of $\lim _{t \rightarrow \infty} u(t)$ follows from Cauchy's criterion and the fact that $\varphi \in \omega(u)$.

By applying Theorem 3 with $\Theta(s)=\frac{1}{C} s^{1-\theta}$ for $\theta \in(0,1)$, we obtain the following corollary.

Corollary 1 Let $M, F$ and $\mathcal{E}$ be as in Theorem 3. Let $u: \mathbb{R}_{+} \rightarrow M$ be a global solution of (1). Assume that there exists $\varphi \in \omega(u), \theta \in(0,1), C \geq 0$ and a neighbourhood $U \subseteq M$ of $\varphi$ such that for every $u \in U$

$$
|\mathcal{E}(v)-\mathcal{E}(\varphi)|^{1-\theta} \leq C\left\langle\mathcal{E}^{\prime}(v), \frac{F(v)}{\|F(v)\|_{g}}\right\rangle
$$

Then $u$ has finite length in $(M, g)$ and, in particular, $\lim _{t \rightarrow+\infty} u(t)=\varphi$ in $(M, g)$.

Note that the Eojasiewicz gradient inequality (4) and the Kurdyka-Lojasiewicz inequality (5) from the Introduction involve only the function $\mathcal{E}$ while the modified Kurdyka-Łojasiewicz inequality (10) and inequality (12) involve in addition the vector field $F$. In fact, the norm of the derivative $\mathcal{E}^{\prime}$ (which appears on the right-hand side in (4) and (5)) is replaced by the directional derivative in the normalized direction of $F$. Let us repeat the two modified inequalities in the following way, that is, from a more geometric point of view:

or

$$
\left\langle(\Theta \circ(\mathcal{E}-\mathcal{E}(\varphi)))^{\prime}, \frac{F}{\|F\|_{g}}\right\rangle \geq 1
$$

$$
\left\langle\left((\mathcal{E}-\mathcal{E}(\varphi))^{\theta}\right)^{\prime}, \frac{F}{\|F\|_{g}}\right\rangle \geq 1 / C
$$


in a neighbourhood of $\varphi$. If $\tilde{g}$ denotes the metric constructed in Theorem 1 , then we can write the inequality (10) also in the form

$$
\Theta(|\mathcal{E}(v)-\mathcal{E}(\varphi)|) \leq \frac{\|F(v)\|_{\tilde{g}}^{2}}{\|F(v)\|_{g}}=\left\|\mathcal{E}^{\prime}(v)\right\|_{\tilde{g}} \frac{\|F(v)\|_{\tilde{g}}}{\|F(v)\|_{g}},
$$

in which the norm of $\mathcal{E}^{\prime}$ with respect to the new metric $\tilde{g}$ and the ratio of the two involved metrics appear. Hence, if the two metrics $g$ and $\tilde{g}$ are equivalent, then (10) reduces to the Kurdyka-Łojasiewicz inequality (5).

As indicated in the Introduction, there are convergence results similar to Theorem 3 in which - instead of the modified Kurdyka-Łojasiewicz inequality - a gradient inequality for the Lyapunov function $\mathcal{E}$ (or an assumption of analyticity) and the angle condition (AC) appear; see [1, Theorem 2.2], [12, Theorem 1.2], [7, Theorem 1] (the latter article considers also the case of differential equations in infinite dimensions). These results (in the case of ordinary differential equations in finite dimensions) can be seen as a consequence of Theorem 3 or its Corollary 1 because the Łojasiewicz inequality (4) and the angle condition (AC) imply the modified Łojasiewicz inequality (12). There are situations, however, in which the modified Łojasiewicz inequality (12) is satisfied but the angle condition (AC) is not. The following elementary example is such a case. A more sophisticated example is described in the following section.

Example 2 Let $M \subseteq \mathbb{R}^{2}$ be the open unit disk, equipped with the Euclidean metric $g$. Let $\alpha \geq 0$, and let $F(u)=F\left(u_{1}, u_{2}\right)=\left(\|u\|^{\alpha} u_{1}-u_{2}, u_{1}+\|u\|^{\alpha} u_{2}\right)$ and $\mathcal{E}(u)=\frac{1}{2}\left(u_{1}^{2}+u_{2}^{2}\right)$. Then

$$
\left\langle\mathcal{E}^{\prime}(u), F(u)\right\rangle=\|u\|^{2+\alpha},\|F(u)\|=\|u\| \cdot \sqrt{1+\|u\|^{2 \alpha}} \text { and }\left\|\mathcal{E}^{\prime}(u)\right\|=\|u\| \text {. }
$$

Hence, unless $\alpha=0$, the angle condition (AC) does not hold on any neighbourhood of the critical point $(0,0)$ (and, by Theorem 2, the Euclidean metric $g$ and the metric $\tilde{g}$ from Theorem 1 are not equivalent). The function $\mathcal{E}$ satisfies the Eojasiewicz inequality (4) near the origin for $\theta=\frac{1}{2}$, but we even have

$$
\frac{1}{\|F(u)\|}\left\langle\mathcal{E}^{\prime}(u), F(u)\right\rangle=\frac{\|u\|^{1+\alpha}}{\sqrt{1+\|u\|^{2 \alpha}}} \geq \frac{1}{\sqrt{2}}\|u\|^{2(1-\theta)} \geq \frac{1}{4} \mathcal{E}(u)^{1-\theta}
$$

provided $0<\theta \leq \frac{1-\alpha}{2}$. Hence, if $0 \leq \alpha<1$, then $\mathcal{E}$ satisfies the modified Łojasiewicz gradient inequality (12).

\section{Application to a second order problem}

Let us consider the following second order problem

$$
\ddot{u}+G(u, \dot{u})+\nabla E(u)=0,
$$


where $E: \mathbb{R}^{N} \rightarrow \mathbb{R}$ and $G: \mathbb{R}^{N} \times \mathbb{R}^{N} \rightarrow \mathbb{R}^{N}$ are two given $C^{2}$ functions. We assume that the second term in this equation represents a damping in the sense that for every $u, v \in \mathbb{R}^{N}$

$$
\begin{aligned}
& \langle G(u, v), v\rangle \geq g(\|v\|)\|v\|^{2}, \\
& \|G(u, v)\| \leq c g(\|v\|)\|v\|, \quad \text { and } \\
& \|\nabla G(u, v)\| \leq c g(\|v\|),
\end{aligned}
$$

where $c \geq 0$ is a constant and $g: \mathbb{R}_{+} \rightarrow \mathbb{R}_{+}$is a nonnegative, concave, nondecreasing function, $g(s)>0$ for $s>0$. Throughout this section, $\|\cdot\|$ denotes the Euclidean norm. The first line in (14) is a lower estimate of the damping in the direction of the velocity.

The following theorem yields convergence of solutions to a singleton.

Theorem 4 Let $u \in W^{2, \infty}\left(\mathbb{R}_{+} ; \mathbb{R}^{N}\right)$ be a global solution of (13). Assume that there exist $\varphi \in \omega(u)$, a neighbourhood $U \subseteq \mathbb{R}^{N}$ of $\varphi$ and a nonnegative, concave, nondecreasing function $\Theta: \mathbb{R}_{+} \rightarrow \mathbb{R}_{+}$such that for every $v \in U$

$$
\Theta(|E(v)-E(\varphi)|) \leq\|\nabla E(v)\|
$$

Assume that $\Theta(s) \leq c \sqrt{s}$ for some $c>0$ and all $s \geq 0$ small enough and that

$$
s \mapsto 1 / \Theta(s) g(\Theta(s)) \in L_{l o c}^{1}([0,+\infty))
$$

Then $u$ has finite length and, in particular, $\lim _{t \rightarrow+\infty} u(t)=\varphi$.

Remark 2 If we take $\Theta(s)=c s^{1-\theta}, G(u, v):=\|v\|^{\alpha} v$ and $g(s):=s^{\alpha}$ for $\alpha \in\left[0, \frac{\theta}{1-\theta}\right), \theta \in\left(0, \frac{1}{2}\right]$, then the equation (13) becomes

$$
\ddot{u}+\|\dot{u}\|^{\alpha} \dot{u}+\nabla E(u)=0
$$

and Theorem 4 applies, thus generalizing a recent convergence result by Chergui (see [6]). However, our theorem allows more general nonlinearities and also growth orders of the damping that are closer to the critical case $\alpha=\frac{\theta}{1-\theta}$. If $\Theta(s)=s^{1-\theta}, g(s)=s^{\theta /(1-\theta)}$ we cannot expect convergence in general as an example by Haraux shows [8]. But if the growth of $\Theta$ or $g$ is a little better, we obtain convergence. For example,

$$
\Theta(s)=s^{1-\theta}, \quad g(s)=s^{\frac{\theta}{1-\theta}} \ln ^{1+\varepsilon}(1 / s)
$$

or

$$
\Theta(s)=s^{1-\theta} \ln ^{1-\theta+\varepsilon}(1 / s), \quad g(s)=s^{\frac{\theta}{1-\theta}}
$$

with $\varepsilon>0$ satisfy the assumptions of Theorem 4 . 
Proof We first show that $\lim _{t \rightarrow \infty}\|\dot{u}(t)\|=0$. Multiplying the equation (13) by $\dot{u}$, integrating from 0 to $t$, and using the assumption on $G$, we obtain

$$
\begin{aligned}
\frac{1}{2}\|\dot{u}(0)\|^{2}+E(u(0)) & =\frac{1}{2}\|\dot{u}(t)\|^{2}+E(u(t))+\int_{0}^{t}\langle G(u(s), \dot{u}(s)), \dot{u}(s)\rangle \mathrm{d} s \\
& \geq \frac{1}{2}\|\dot{u}(t)\|^{2}+E(u(t))+\int_{0}^{t} g(\|\dot{u}(s)\|)\|\dot{u}(s)\|^{2} \mathrm{~d} s .
\end{aligned}
$$

Since $u$ and $\dot{u}$ are globally bounded, this implies

$$
\int_{0}^{\infty} g(\|\dot{u}(s)\|)\|\dot{u}(s)\|^{2} \mathrm{~d} s<+\infty .
$$

Since, moreover, $\dot{u}$ is uniformly continuous, we obtain that $\lim _{t \rightarrow \infty} g(\|\dot{u}(s)\|)\|\dot{u}(s)\|^{2}=$ 0 . Since $g(s)>0$ whenever $s>0$, this implies that $\lim _{t \rightarrow \infty}\|\vec{u}(t)\|=0$.

Note that $u$ is a (global) solution of (13) if and only if $(u, \dot{u})$ is a (global) solution to the following problem

$$
\frac{d}{d t}\left(\begin{array}{l}
u(t) \\
v(t)
\end{array}\right)+F\left(\begin{array}{l}
u(t) \\
v(t)
\end{array}\right)=0
$$

where

$$
F(u, v)=\left(\begin{array}{c}
-v \\
G(u, v)+\nabla E(u)
\end{array}\right) .
$$

Let $M \subseteq \mathbb{R}^{N} \times \mathbb{R}^{N}$ be a suffiently large (closed) ball which is a neighbourhood of the range of $(u, \dot{u})$. Note that, by the above argument and by assumption, $(\varphi, 0)$ belongs to the $\omega$-limit set of $(u, \dot{u})$. In the following we will often use boundedness of continuous functions on $M$, in particular there exists a constant $K$ such that

$$
g(\|v\|),\|G(u, v)\|,\|\nabla G(u, v)\|,\|\nabla E(u)\| \leq K
$$

and

$$
g(\|\nabla E(u)\|),\|G(u, \nabla E(u))\|,\|\nabla G(u, \nabla E(u))\| \leq K
$$

for all $(u, v) \in M$.

Following the idea of the proof by Chergui [6], we define, for $\varepsilon>0$ small and to be chosen below,

$$
\mathcal{E}(u, v):=\frac{1}{2}\|v\|^{2}+E(u)+\varepsilon\langle G(u, \nabla E(u)), v\rangle .
$$

We show that $\mathcal{E}$ is a strict Lyapunov function for (15). We compute

$$
\begin{aligned}
\left\langle\mathcal{E}^{\prime}(u, v), F(u, v)\right\rangle= & \langle G(u, v), v\rangle-\varepsilon\left\langle\nabla G(u, \nabla E(u))\left(I d, \nabla^{2} E(u)\right) v, v\right\rangle+ \\
& +\varepsilon\langle G(u, \nabla E(u)), G(u, v)\rangle+\varepsilon\langle G(u, \nabla E(u)), \nabla E(u)\rangle
\end{aligned}
$$


By the assumption on $G$ (first line of (14)), we have

$$
\begin{aligned}
\langle G(u, v), v\rangle & \geq g(\|v\|)\|v\|^{2} \text { and } \\
\varepsilon\langle G(u, \nabla E(u)), \nabla E(u)\rangle & \geq \varepsilon g(\|\nabla E(u)\|)\|\nabla E(u)\|^{2} .
\end{aligned}
$$

By the second line of (14) and by Cauchy-Schwarz, for every $(u, v) \in M$ we can estimate

$$
\begin{aligned}
|\varepsilon\langle G(u, \nabla E(u)), G(u, v)\rangle| & \leq c^{2} \varepsilon g(\|\nabla E(u)\|)\|\nabla E(u)\| g(\|v\|)\|v\| \\
& \leq \frac{1}{4} \varepsilon g(\|\nabla E(u)\|)\|\nabla E(u)\|^{2}+C \varepsilon g(\|v\|)\|v\|^{2} .
\end{aligned}
$$

Here and in the following, $C \geq 0$ denotes a constant which may change from line to line, which depends on $K$, but which is independent from $\varepsilon>0$. Again by using the assumptions on $G$, by using that $g$ is nondecreasing, and by Lemma 1 (b), we obtain that for every $(u, v) \in M$

$$
\begin{aligned}
& \mid \varepsilon\langle\nabla G\left.(u, \nabla E(u))\left(I d, \nabla^{2} E(u)\right) v, v\right\rangle \mid \leq \\
& \leq C \varepsilon g(\|\nabla E(u)\|)\|v\|^{2} \\
& \quad \leq \begin{cases}\frac{1}{4} \varepsilon g(\|\nabla E(u)\|)\|\nabla E(u)\|^{2} & \text { if } 2 \sqrt{C}\|v\| \leq\|\nabla E(u)\|, \\
C \varepsilon g(2 \sqrt{C}\|v\|)\|v\|^{2} & \text { if } 2 \sqrt{C}\|v\| \geq\|\nabla E(u)\|\end{cases} \\
& \quad \leq C \varepsilon g(\|v\|)\|v\|^{2}+\frac{1}{4} \varepsilon g(\|\nabla E(u)\|)\|\nabla E(u)\|^{2} .
\end{aligned}
$$

Taking the preceding estimates together, we obtain for every $(u, v) \in M$

$$
\left.\left\langle\mathcal{E}^{\prime}(u, v), F(u, v)\right\rangle \geq(1-2 C \varepsilon) g(\|v\|)\|v\|^{2}+\frac{1}{2} \varepsilon g(\|\nabla E(u)\|)\|\nabla E(u)\|^{2}\right) .
$$

In particular, for $\varepsilon>0$ small enough, $\mathcal{E}$ is a strict Lyapunov function for the problem (15) on $M$. From the preceding estimate, from the estimate

$$
\|F(u, v)\| \leq C(\|v\|+\|\nabla E(u)\|),
$$

and from the estimate

$$
\begin{gathered}
(\|v\|+\|\nabla E(u)\|)(g(\|v\|)\|v\|+g(\|\nabla E(u)\|)\|\nabla E(u)\|) \leq \\
3\left(g(\|v\|)\|v\|^{2}+g(\|\nabla E(u)\|)\|\nabla E(u)\|^{2}\right)
\end{gathered}
$$

(Lemma 2), we obtain the lower estimate

$$
\frac{1}{\|F(u, v)\|}\left\langle\mathcal{E}^{\prime}(u, v), F(u, v)\right\rangle \geq \alpha(g(\|v\|)\|v\|+g(\|\nabla E(u)\|)\|\nabla E(u)\|)
$$

on $M$, where $\alpha>0$ is a constant depending only on uniform bounds of the functions $E, G$ and $g$. 
Next, by the definition of $\mathcal{E}$ and by the assumptions on $G$,

$$
\begin{aligned}
|\mathcal{E}(u, v)-\mathcal{E}(\varphi, 0)| & \leq \frac{1}{2}\|v\|^{2}+|E(u)-E(\varphi)|+\varepsilon c g(\|\nabla E(u)\|)\|\nabla E(u)\|\|v\| \\
& \leq\|v\|^{2}+|E(u)-E(\varphi)|+C \varepsilon\|\nabla E(u)\|^{2} .
\end{aligned}
$$

By Lemma 1 (applied with the function $h=\Theta$ )

$$
\Theta(|\mathcal{E}(u, v)-\mathcal{E}(\varphi, 0)|) \leq \Theta\left(\|v\|^{2}\right)+\Theta(|E(u)-E(\varphi)|)+C \Theta\left(\|\nabla E(u)\|^{2}\right) .
$$

By the assumption on $\Theta$ and by the gradient inequality for $E$, we obtain that there exists a neighbourhood $\mathcal{U} \subseteq M$ of $(\varphi, 0)$ such that for every $(u, v) \in \mathcal{U}$

$$
\Theta(|\mathcal{E}(u, v)-\mathcal{E}(\varphi, 0)|) \leq C(\|v\|+\|\nabla E(u)\|) .
$$

Now let $\tilde{\Theta}: \mathbb{R}_{+} \rightarrow \mathbb{R}_{+}$be given by $\tilde{\Theta}(s):=\Theta(s) g(\Theta(s))$. By Lemmas 1 and 2 , we then have

$$
\begin{aligned}
\tilde{\Theta}(|\mathcal{E}(u, v)-\mathcal{E}(\varphi, 0)|) & \leq C(\|v\|+\|\nabla E(u)\|) g(\|v\|+\|\nabla E(u)\|) \\
& \leq C(g(\|v\|)\|v\|+g(\|\nabla E(u)\|)\|\nabla E(u)\|)
\end{aligned}
$$

Taking this estimate and (17) together, we have proved that there exists a neighbourhood $\mathcal{U} \subseteq \mathbb{R}^{N} \times \mathbb{R}^{N}$ of $(\varphi, 0)$ such that for every $(u, v) \in \mathcal{U}$

$$
\tilde{\Theta}(|\mathcal{E}(u, v)-\mathcal{E}(\varphi, 0)|) \leq C\left\langle\mathcal{E}^{\prime}(u, v), \frac{F(u, v)}{\|F(u, v)\|}\right\rangle .
$$

Moreover, by assumption, $1 / \tilde{\Theta} \in L_{l o c}^{1}([0, \infty))$. Hence, Theorem 3 can be applied and $\lim _{t \rightarrow \infty}(u(t), \dot{u}(t))=(\varphi, 0)$.

Remark 3 If $G(u, v)=\|v\|^{\alpha} v$ for some $\alpha \geq 0$ and if $g(v)=\|v\|^{\alpha}$, then one easily checks that the lower estimate (16) is optimal. Moreover, the estimate $\left\|\mathcal{E}^{\prime}(u, v)\right\|+\|F(u, v)\| \leq C(\|v\|+\|\nabla E(u)\|)$ is optimal for small $v$ and $\nabla E(u)$. Hence, if $\alpha>0$, then $\mathcal{E}^{\prime}$ and $F$ do not satisfy the angle condition (AC).

Lemma 1 Let $h: \mathbb{R}_{+} \rightarrow \mathbb{R}_{+}$be a nonnegative, concave, nondecreasing function. Then:

(a) For every $u, v \geq 0$ one has $h(u+v) \leq h(u)+h(v)$.

(b) For every $C>0$ and every $u \geq 0$ one has $h(C u) \leq \max \{C, 1\} h(u)$.

Proof (a) By concavity we have the inequalities

$$
\begin{aligned}
& \frac{u}{u+v} h(u+v)+\frac{v}{u+v} h(0) \leq h(u) \quad \text { and } \\
& \frac{v}{u+v} h(u+v)+\frac{u}{u+v} h(0) \leq h(v) .
\end{aligned}
$$

Summing up the two inequalities and using that $h(0) \geq 0$ yields the desired result.

(b) If $C \leq 1$, the inequality follows from the assumption that $h$ is nondecreasing. If $\bar{C} \geq 1$, the concavity and the assumption $h(0) \geq 0$ imply

$$
\frac{1}{C} h(C u) \leq \frac{1}{C} h(C u)+\frac{C-1}{C} h(0) \leq h(u) .
$$


Lemma 2 Let $r, s \in C^{1}\left(\mathbb{R}_{+}\right)$be nonnegative and nondecreasing. Then, for every $u, v \geq 0$,

$$
r(u) s(v) \leq r(u) s(u)+r(v) s(v) .
$$

Proof For fixed $u \geq 0$, consider the function $v \mapsto r(u) s(u)+r(v) s(v)-r(u) s(v)$. Observe first that this function is nonnegative for $v \leq u$, since $r$ and $s$ are nonnegative and $s$ is nondecreasing. Then observe that the derivative of this function is nonnegative for $v \geq u$ by the assumptions on $r$ and $s$.

\section{Acknowledgements}

The work on this article started while the first author visited the Paul Verlaine University of Metz. He would like to thank Eva Fašangová and Ralph Chill for their kind hospitality during his stay in Metz.

\section{References}

1. P.-A. Absil, R. Mahony, and B. Andrews, Convergence of the iterates of descent methods for analytic cost functions, SIAM J. Optim. 16 (2005), 531-547 (electronic).

2. F. Alvarez, H. Attouch, J. Bolte, and P. Redont, A second-order gradient-like dissipative dynamical system with Hessian driven damping: application to optimization and mechanics, J. Math. Pures Appl. 81 (2002), 747-779.

3. N. P. Bhatia and G. P. Szegö, Stability Theory of Dynamical Systems, Springer Verlag, Berlin, Heidelberg, New York, 1970.

4. J. Bolte, A. Daniilidis, A. Lewis, and M. Shiota, Clarke subgradients of stratifiable functions, SIAM J. Optim. 18 (2007), no. 2, 556-572 (electronic).

5. J. Bolte, A. Daniilidis, O. Ley, and L. Mazet, Characterizations of Eojasiewicz inequalities: subgradient flows, talweg, convexity, Trans. Amer. Math. Soc. 362 (2010), no. 6, 3319-3363.

6. L. Chergui, Convergence of global and bounded solutions of a second order gradient like system with nonlinear dissipation and analytic nonlinearity, J. Dynam. Differential Equations 20 (2008), no. 3, 643-652.

7. R. Chill, A. Haraux, and M. A. Jendoubi, Applications of the Eojasiewicz-Simon gradient inequality to gradient-like evolution equations, Anal. Appl. 7 (2009), 351-372.

8. A. Haraux, Systèmes dynamiques dissipatifs et applications, Masson, Paris, 1990.

9. A. Haraux and M. A. Jendoubi, Convergence of solutions to second-order gradient-like systems with analytic nonlinearities, J. Differential Equations 144 (1998), 313-320.

10. S.-Z. Huang, Gradient Inequalities: with Applications to Asymptotic Behaviour and Stability of Gradient-like Systems, Mathematical Surveys and Monographs, vol. 126, Amer. Math. Soc., Providence, R.I., 2006.

11. K. Kurdyka, On gradients of functions definable in o-minimal structures, Ann. Inst. Fourier (Grenoble) 48 (1998), 769-783.

12. Ch. Lageman, Pointwise convergence of gradient-like systems, Math. Nachr. 280 (2007), no. 13-14, 1543-1558.

13. J. P. Lasalle, Asymptotic stability criteria, Proc. Symp. Appl. Math., Vol. XIII, American Mathematical Society, Providence, R.I., 1962, pp. 299-307.

14. A. M. Liapunov, Stability of motion, With a contribution by V. A. Pliss and an introduction by V. P. Basov. Translated from the Russian by Flavian Abramovici and Michael Shimshoni. Mathematics in Science and Engineering, Vol. 30, Academic Press, New York, 1966.

15. S. Łojasiewicz, Une propriété topologique des sous-ensembles analytiques réels, Colloques internationaux du C.N.R.S.: Les équations aux dérivées partielles, Paris (1962), Editions du C.N.R.S., Paris, 1963, pp. 87-89. 
16. R. I. McLachlan, G. R. W. Quispel, and N. Robidoux, Unified approach to Hamiltonian systems, Poisson systems, gradient systems, and systems with Lyapunov functions or first integrals, Phys. Rev. Lett. 81 (1998), no. 12, 2399-2403.

17. R. I. McLachlan, G. R. W. Quispel, and N. Robidoux, Geometric integration using discrete gradients, R. Soc. Lond. Philos. Trans. Ser. A Math. Phys. Eng. Sci. 357 (1999), no. 1754, 1021-1045. 